\title{
High-Spatial and High-Energy Resolution EELS Studies of Chemical and Electronic Properties of Interfaces and Nanostructures
}

\author{
K.J. Dudeck ${ }^{1}$, M. Couillard ${ }^{1}$, N. Gauquelin ${ }^{1}$, L. Gunawan ${ }^{1}$, S. Hosseini Vajargah ${ }^{1}$, S. Lazar ${ }^{1,2}$, D. \\ Rossouw ${ }^{1}$, Y. Shao ${ }^{1}$, G. Zhu' ${ }^{1}$, G.A. Botton ${ }^{1}$ \\ 1 Department of Materials Science and Engineering and Canadian Centre for Electron Microscopy, \\ McMaster University, 1280 Main St. W., ON, Canada, L8S 4M1 \\ 2 FEI Electron Optics, 5600 KA Eindhoven, The Netherlands
}

The development of aberration-corrected transmission electron microscopes capable of high-energy resolution Electron Energy Loss Spectroscopy (EELS) has enabled the study of the chemical composition of materials at unprecedented spatial resolution together with the prospects of providing new insight onto the electronic properties of such materials. Elemental maps demonstrating atomic resolution, even at low-accelerating voltages, have been established recently $[1,2]$. Such experiments open the path to the study of complex electronic structure effects occurring in materials such as complex oxides, interfaces between films and their substrates and grain boundaries. Although there is significant interest on such studies, there are still several challenges that need to be overcome when detailed structural and electronic information is to be retrieved. This contribution presents examples of applications of high-spatial and high-energy resolution EELS studies and the quantitative analysis of the data in order to highlight information retrieved as well as the pitfalls and the difficulties in the interpretation.

Experiments were carried out on an FEI Titan 80-300 Cubed equipped with aberration-correctors of the probe-forming lens and imaging lens as well as a monochromator and a high-brightness source. The system is located in an ultra-stable environment allowing the reliable quantitative measurement of strain on scanning-transmission electron microscopy images[3]. Examples of EELS elemental maps on grain boundaries in bicrystals of $\mathrm{SrTiO}_{3}$ have shown the detection of depletion of $\mathrm{Sr}$ atoms within one unit cell from the grain boundary (figure 1). Quantitative elemental maps have also been processed with and without the use of multivariate statistical analysis methods in order to understand the effect of signal processing parameters on the information content of images. Detailed analysis using measurements of signal-to-noise ratios (SNR) have been performed and show that the improvements in the quality of the images are related to the reduction of the variance in the SNR values. Such measurements have been carried out with an in-house parallelized MATLAB code capable of processing spectrum images. Automated extraction of the EELS signal based on optimization of the SNR (such as shown figure 1d) and as a function of the number of components in the statistical analysis has been developed [4]. The quantification of the spectrum-images and the measurement of energies of the edges have shown that changes in the valence and core-level shift have been detected within less than $1 \mathrm{~nm}$ form the boundary centre consistent with the local change in composition and local charge. In the case of interfaces in ferroelectric $\mathrm{Bi}_{3.25} \mathrm{La}_{0.75} \mathrm{Ti}_{3} \mathrm{O}_{12}$ deposited on $\mathrm{SrRuO}_{3} / \mathrm{SrTiO}_{3}$, we have shown that the apparently sharp interfaces, as assessed by high-angle annular dark-field (HAADF) STEM, are, in fact, chemically diffuse with atomic steps on only one of the cationic sublattices hence suggesting that the EELS mapping approach can even reveal changes in the surface termination of substrates (figure 2) and thus provide key information on the growth mechanisms in thin films and the origin of defects. Examples of the application of high-resolution 
EELS in the low-loss region and core-loss region applied to the study of optical fluorescent/chromophore probes, and the valence changes at interface will be highlighted [5].

\section{References}

[1] S. Lazar et al., Microscopy and Microanalysis, 16, 416-424, (2010)

[2] G. Botton, S. Lazar, C. Dwyer, Ultramicroscopy, 110, 926-934(2010)

[3] S. Hosseini Vajargah, et al. Applied Physics Letters, 98, (2011), doi:10.1063/1.3551626

[4] K.J. Dudeck et al, Submitted to Micron 2011.

[5] This research was supported by NSERC. The work was carried out at the Canadian Centre for Electron Microscopy, a facility supported by NSERC and McMaster University.

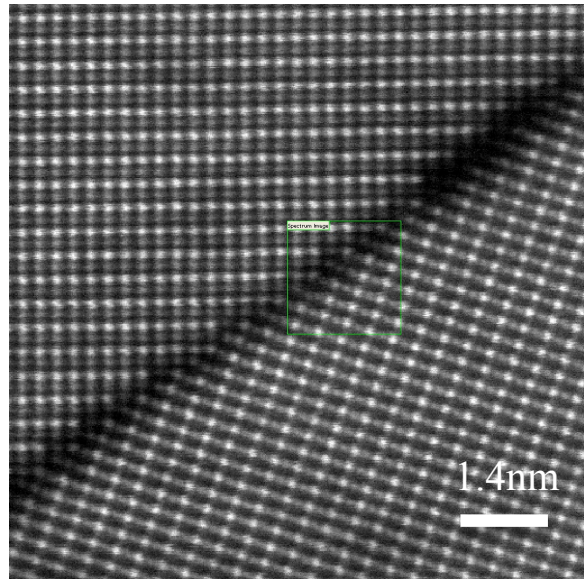

1a)

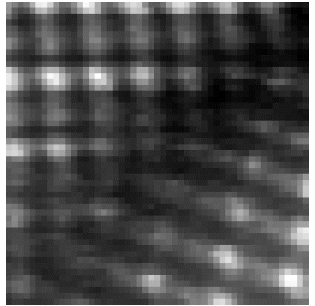

1b)

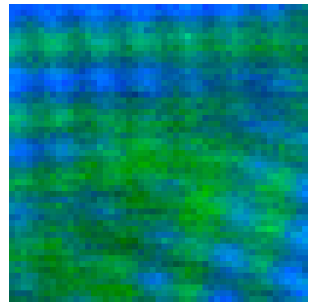

1c)

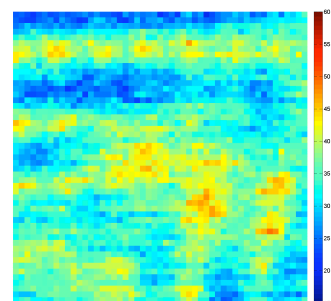

1d)

Figure 1. a) HAADF STEM image of the grain boundary in $\mathrm{SrTiO}_{3}$ with selected area of interest, b) HAADF signal on the spectrum image dataset, c) elemental maps of Ti (green) and $\mathrm{Sr}$ (blue). 1d) Signal to noise ratio (SNR) map for the Ti edge calculated with a parallelized MATLAB code developed to optimize the SNR in the images [4].

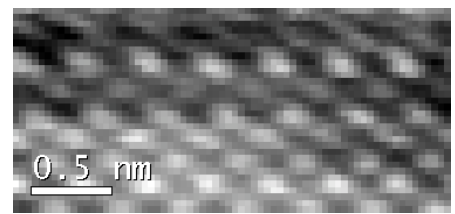

2b) $\mathrm{ADF}$

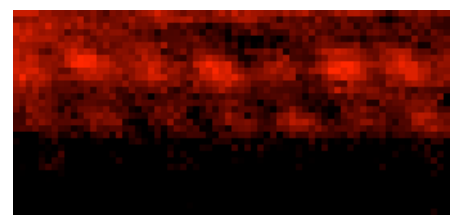

2c) $\mathrm{La}$

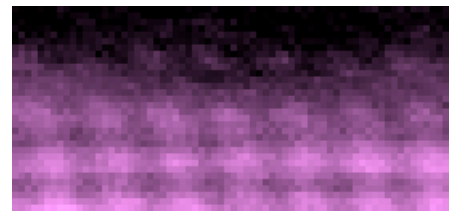

2d) $\mathrm{Sr}$

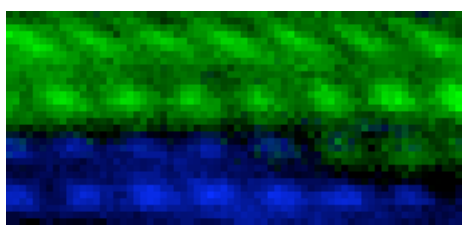

2e) composite $\mathrm{Ti} / \mathrm{Ru}$ map

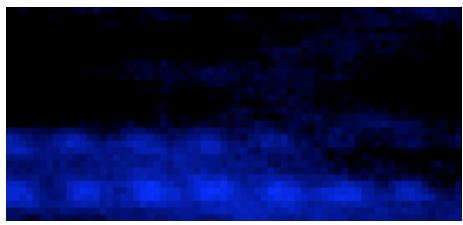

2f) $\mathrm{Ru}$

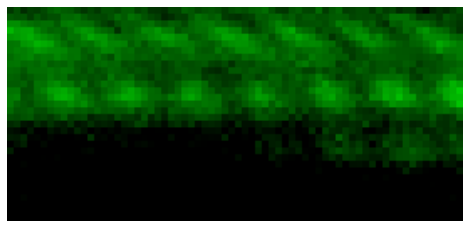

2g) $\mathrm{Ti}$

2a)

Figure 2. Interface between $\mathrm{SrRuO}_{3}$ film (grown on $\mathrm{SrTiO}_{3}$ ) and $\mathrm{Bi}_{3.25} \mathrm{La}_{0.75} \mathrm{Ti}_{3} \mathrm{O}_{12}$. Interface region a) and HAADF signal on the spectrum image b), and elemental maps of La 2c), Sr 2d), composite map of Ru (blue) and Ti (green), elemental maps of Ru 2f) and Ti 2g). 